\title{
Differenzierung, Dezentralisierung, Ambulantisierung
}

\section{Die wichtigsten Veränderungen in der Sozialwirtschaft haben massiven Einfluss auf Personalfragen}

\author{
ATTILA NAGY UND \\ CARSTEN EFFERT \\ Der Arzt und Diplom-Volkswirt \\ Attila Nagy ist seit mehr als 20 Jah- \\ ren beratend in der Sozialwirtschaft \\ tätig. Im Jahre 1997 gründete er \\ zusammen mit Dr. Michael Rosen- \\ baum die "rosenbaum nagy unter- \\ nehmensberatung“. Der gebürtige \\ Ungar beschäftigt sich schwer- \\ punktmäßig mit den Themenfel- \\ dern Strategie, (Re-) Organisation, \\ Krisenmanagement. Er ist auch als \\ Interimsmanager tätig.
}

www.rosenbaum-nagy.de

Carsten Effert ist Diplom-Ökonom und Diplom-Sozialpädagoge und seit acht Jahren bei der rosenbaum nagy unternehmensberatung in Köln tätig. Dort beschäftigt er sich als Seniorberater schwerpunktmäßig mit den Themenfeldern Strategie, Geschäftsfeldentwicklung und (Re-) Organisation in der Sozialwirtschaft.

www.rosenbaum-nagy.de

\author{
Die sozialen Organisationen und Unternehmen stehen \\ vor großen Herausforderungen, vom demografischen \\ Wandel bis zu neuen Erwartungen von den Nutzern, \\ dem Gesetzgeber und der Öffentlichkeit. Die \\ Fachkräfte müssen diesen Anforderungen flexibel \\ begegnen und ihre neuen Rollen annehmen.
}

Personalfragen - also alle Fragen hinter der Leitfrage, welche Mitarbeitenden wo, wie und wie lange eingesetzt werden - sind seit jeher von zentraler Bedeutung in der Sozial- und Gesundheitswirtschaft. Denn einerseits wird die erbrachte Dienstleistung stark durch persönliche Leistungen der Mitarbeitenden geprägt und andererseits stellen die Personalkosten die wichtigste Kostenposition dar, die es zu steuern gilt.

Umso erstaunlicher ist die Tatsache, dass trotz aller Bekenntnisse die Personalarbeit in der Sozialwirtschaft lange Zeit ein Schattendasein fristete und bei vielen Trägern bis heute nicht die eigentlich erforderliche Bedeutung erlangt hat.

Lange Zeit beschränkten sich die personalbezogenen Sachverhalte vor allem darauf, wie das zumeist ausreichend vorhandene Personal bestmöglich eingesetzt werden konnte, damit es den betreuten Menschen gut dabei ging. Eine hohe fachliche Qualität und damit ein hoher Professionalisierungsgrad wurden seitens der Verbände eingefordert und zumeist auch erfolgreich gegenüber den Kostenträgern durchgesetzt.

Von den Mitarbeitenden wurden aufgrund suboptimaler Planungsprozesse teilweise hohe Flexibilitäten abgefordert. Zugleich mutete man aber auch den betreuten Menschen immer wieder Zugeständnisse zu, indem sie sich an die Institution anzupassen hatten, so dass insbesondere im stationären Setting die
Strukturen und Abläufe oft stärker mitarbeiterorientiert als kundenorientiert waren.

Seit einigen Jahren vollziehen sich allerdings in mehreren Bereichen grundlegende Veränderungen in der Branche, die sowohl in der strategischen Personalarbeit als auch in der Steuerung des Personaleinsatzes neue Herausforderungen mit sich bringen:

- die Ausgaben explodieren und müssen begrenzt werden

- den Hilfebedarfen muss individueller und kundenorientierter entsprochen werden

- die Wirkung der Arbeit muss zunehmend nachgewiesen werden

- fehlendes Fachpersonal stellt immer öfter ein zentrales Wachstumshemmnis dar

Es braucht daher neue Konzepte, wie und vor allem von wem die zu betreuenden Menschen zukünftig versorgt werden. Die Qualität der Personalarbeit wird ein zunehmend wichtiger Wettbewerbsfaktor.

\section{Die Grenzen des Wachstums}

Zwar hört man seit Jahren die Klagen über Kürzungen im Sozialbereich, faktisch sind die Kosten in vielen Marktsegmenten in den letzten Jahren jedoch geradezu explodiert. Besonders hervorzuheben sind hier - aufgrund der demo- 
grafischen Entwicklung - die Altenhilfe sowie - aufgrund des U3-Ausbaus und der steigenden Zahl an Inobhutnahmen - die Kinder- und Jugendhilfe.

Zwar stehen dem sowohl signifikante Kürzungen der Gesamtetats in einigen anderen Bereichen, beispielsweise der Beschäftigungsförderung, als auch Kürzungen auf Einzelfallebene gegenüber, dennoch befindet sich insgesamt mehr Geld »im System «.

Da es zukünftig immer weniger Einzahler ins Steuer- und Sozialversicherungssystem gibt, ist davon auszugehen, dass es in den nächsten Jahren und Jahrzehnten zu Ausgabenkürzungen kommen wird. Auch wenn dies möglicherweise nicht für die Gesamtausgaben gelten wird, so ist jedoch davon auszugehen, dass zumindest die Durchschnittskosten je Fall reduziert werden. Es stellt sich hierbei die Frage, welche Effizienzreserven im System gehoben werden können, um Qualitätseinschränkungen für die zu versorgenden Menschen möglichst zu vermeiden oder in welchen Bereichen welche Qualitätseinbußen der Versorgung am ehesten zu verschmerzen sind.

Oft gibt es bei den Sachkosten noch Reserven, die beispielsweise durch eine Professionalisierung des Einkaufs oder eine Reduktion von Standards zum Beispiel in der hauswirtschaftlichen Versorgung erschlossen werden könnten. Aufgrund des hohen Anteils an den Gesamtkosten spielt eine Optimierung der Personalkosten eine zentrale Rolle. Hier sind folgende Ansätze besonders relevant:

- deutliche Verbesserung der PersonalFeinsteuerung, insbesondere durch eine genauere Anpassung des Personaleinsatzes an die Nachfragesituation

- Reduktion des Professionalisierungsgrades, wo dies möglich ist

- gegebenenfalls die Entwicklung alternativer Versorgungskonzepte mit einer geringeren Personalintensität

Während bei den ersten beiden Punkten bereits bei den bestehenden Geschäftsund Refinanzierungsmodellen einige Spielräume bestehen, geht es bei dem dritten Punkt auch um die Entwicklung neuer Versorgungsformen, die sowohl technische als auch organisatorische Innovationen erfordern.
Insgesamt ist in der Perspektive davon auszugehen, dass sich die Art der Betreuung der Menschen verändern wird. Der Anteil an Fachkräften wird deutlich sinken (müssen), und auch Umfang und Häufigkeit der persönlichen Versorgung durch Profis werden sich tendenziell reduzieren. Eine Kompensation kann einerseits durch eine Erhöhung von Selbstzahlerleistungen erfolgen, soweit die zu versorgenden Menschen oder ihre Angehörigen hierzu finanziell in der Lage sind. Andererseits werden Laienhelfer, Netzwerke oder der Einsatz technischer Hilfsmittel eine zunehmende Rolle spielen. Hierbei verändert sich die Aufgabe der Fachkräfte von der unmittelbaren Leistungsausführung hin zur Organisation und Koordination der Leistungen. Eine Aufgabe wird hierbei möglicherweise in der Priorisierung und Rationierung von Leistungen bestehen.

\section{Individualisierung der Angebote}

Die beschriebene Kostenentwicklung resultiert im Wesentlichen aus einem steigenden Hilfebedarf. Zum einen ist in vielen Teilbranchen ein quantitatives Wachstum zu beobachten, wie beispielsweise in der Altenhilfe. Zum anderen gibt es aber auch ein qualitatives Wachstum. Dies gilt zum Beispiel für die Kinder- und Jugendhilfe, denn hier findet neben dem quantitativen Wachstum aus dem U3-Ausbau auch zunehmend ein qualitatives statt: Der Hilfebedarf im Einzelfalle und die Anzahl der extremen »Problemfälle « steigen. Außerdem spielt ein verändertes Verständnis von Krankheiten (z. B. Autismus, ADHS) eine treibende Rolle.

Gleichzeitig steigen die Anforderungen seitens der Kostenträger. Mit Leitkonzepten, wie Sozialraumorientierung, Inklusion und der Personenzentrierung verbindet sich ein Paradigmenwechsel: Nicht länger steht die Vorhaltung bestimmter Hilfestrukturen im Mittelpunkt, sondern die individualisierte Hilfe.

Parallel ist eine zunehmende Emanzipation der Kunden wahrzunehmen. Zwar bleibt die Sozial- und Gesundheitswirtschaft eine eher komplexe und intransparente Branche, aber in Zeiten des Internets stehen vielen Hilfebedürftigen und ihren Angehörigen deutlich mehr Informationen zur Verfügung als früher. Daneben gibt es spezielle Beratungsstellen und Aufklärung durch die Kostenträger. Insgesamt führt dies dazu, dass die betroffenen Menschen heute viel genauer wissen, was ihnen zusteht, wie sie die Hilfe erhalten können und welche Leistungen sie gegebenenfalls als Selbstzahler "hinzubuchen " können.

All dies führt zu einer Individualisierung der Hilfen, verbunden mit einer Differenzierung, Dezentralisierung und Ambulantisierung der Angebote. Dies bedeutet nicht weniger als einen Paradigmenwechsel. Wo früher die Menschen in großen Leistungseinheiten, wie den klassischen stationären Behindertenwohneinrichtungen, mit Standardleistungen versorgt wurden (Wohnen, Freizeit, Arbeit), werden heute stationäre Einrichtungen abgebaut und Angebote ambulantisiert. Dies bringt deutliche Veränderungen für den Personaleinsatz mit sich: Die Mitarbeitenden müssen dort sein, wo die Menschen sind - und zwar genau zu den Zeiten, an denen sie da sind. Das mag sich profan anhören, entspricht aber noch nicht überall der Realität. Ein so veränderter Personaleinsatz bedeutet eine andere Dienstplanung, andere Arbeitsorte und in der Regel einen anderen Qualifikationsmix.

Eine Individualisierung und Ambulantisierung von Leistungen stellt sehr hohe Anforderungen an die Personalsteuerung, da die Leistungen kleinteiliger werden und der Auftragsbestand deutlicheren Schwankungen unterliegt. Dies bedeutet eine enge auftragsbezogene Steuerung mit der notwendigen Flexibilisierung des Personaleinsatzes. Für die in vielen Segmenten stark stationär sozialisierten Mitarbeiterinnen und Mitarbeiter bedeutet das eine erhebliche Umstellung und für die Träger die Notwendigkeit, hierfür organisatorische Grundlagen zu schaffen. $\mathrm{Zu}$ diesen gehören insbesondere flexible Arbeitszeitmodelle, Arbeitszeitkonten sowie Feinsteuerungsinstrumente.

\section{Die Wirkung macht's}

In den skizzierten Kontext passt der aktuelle Versuch vieler Kostenträger, die Wirkung der Hilfeerbringung stärker in den Fokus zu nehmen. Bekanntestes Beispiel dürfte der sogenannte »Wirkungsdialog « in der Kinder- und 
Jugendhilfe sein. Aber auch viele Träger entdecken die Wirkungsorientierung als Mittel der Lobbyarbeit für sich und passen ihr Leistungscontrolling dem an. Damit stehen neben dem bislang fokussierten »Output « (also die quantitative Leistungsmenge) inzwischen auch die Dimensionen »Effect « (objektiver Effekt), »Impact « (subjektiver Effekt auf den Leistungsempfänger) und »Outcome« (gesellschaftlicher Nutzen) im Mittelpunkt der Betrachtung.

Diese Entwicklung kann in konsequenter Anwendung zu einem Innovationsschub in der Sozialwirtschaft führen. Neben technischen Innovationen (z. B. AAL, Telemedizin, Einsatz von multimedialer Kommunikationsmitteln) geht es insbesondere um die Schaffung neuer Versorgungsstrukturen mit einer veränderten Arbeitsteilung.

Neben Fachkräften, die weiterhin benötigt werden, nicht zuletzt bei steigendem qualitativen Hilfebedarf, werden zunehmend auch Nicht-Fachkräfte oder Laienhelfer und Netzwerke gebraucht. Diese sind bei der deutlich aufwendigeren, weil individualisierten Hilfeerbringung für die Fachkräfte eine wertvolle und zudem oftmals günstigere Ergänzung - und zwar ohne Qualitätseinbußen hinsichtlich der erreichten Wirkung. Insbesondere Konzepte der Sozialraumorientierung wird damit die Wirkung der Leistung deutlich in den Mittelpunkt gestellt. Man geht, mit guten Gründen, davon aus, dass eine individualisierte Hilfeerbringung in einem familienähnlichen Umfeld höhere Erfolgsaussichten hat.

Eine zentrale Aufgabe der professionellen Helfer wird zukünftig darin liegen, mobilisierbare Ressourcen zu identifizieren und bestmöglich zu organisieren, um mit einem begrenzten Input eine gute individuelle Problemlösung zu bewirken. Dies beinhaltet auch eine individuell festzulegende und auszugestaltende Betreuungsintensität. Hierbei müssen kreative Konzepte entwickelt werden; eine Übertragung stationärer Versorgungskonzepte auf den ambulanten Bereich wird keinen Erfolg haben.

\section{Fachkräftemangel als zentrales Entwicklungshemmnis}

Eine ähnlich starke Rolle der Fachkräfte in der unmittelbaren Leistungserbringung wie bislang wird zukünftig aufgrund des vielfach um sich greifenden Fachkräftemangels auch gar nicht mehr möglich sein. Was in der Pflege begann und dort inzwischen flächendeckenden Charakter angenommen hat, ist auch in anderen Branchen inzwischen ein Problem. Vor allem Er-

\section{"Träger scheuen inzwischen das Risiko, neue Einrichtungen zu eröffnen, weil sie befürchten, kein geeignetes Personal zu finden«}

sowie der Personenzentrierung setzen auf die Aktivierung der eigenen Ressourcen sowie die Potenziale von Netzwerken, wodurch das Leben in künstlichen Parallelwelten zugunsten einer echten und dauerhaften Teilhabe zurückgedrängt wird.

Zudem steigen die fachlichen Anforderungen der Kostenträger. Neben der Unterbringung in Pflegefamilien gewinnt beispielsweise die gemeinsame Wohngemeinschaft mit einer »Bezugserzieherin« als Modell der Versorgung von Kindern und Jugendlichen zunehmend an Bedeutung. Ein Stück weit unabhängig von möglichen Kostenfragen zieher und Heilerziehungspfleger sind in bestimmten Regionen Deutschlands inzwischen dringend gesuchte Mitarbeitende.

Es kommt mittlerweile immer öfter vor, dass Einrichtungen später oder nicht vollständig eröffnet werden können, nicht etwa weil die Kunden, sondern weil die Mitarbeiter fehlen. Träger scheuen teilweise das Risiko, neue Einrichtungen zu eröffnen, weil sie befürchten, kein geeignetes Personal zu finden.

Damit ist das Personal heute einer der wichtigsten Veränderungstreiber in der Branche. Die Kräfteverhältnisse auf dem Arbeitsmarkt haben sich deutlich verschoben. Die Träger müssen einiges tun, um geeignetes Personal in ausreichendem Maße zu finden. Hinzu kommen die Auswirkungen alternder Belegschaften mit einer höheren Anfälligkeit für Krankheiten und eingeschränkter physischer Belastbarkeit. Und die jüngeren Mitarbeiterinnen und Mitarbeiter kommen mit anderen Vorstellungen ins Berufsleben und sind eher bereit, den Arbeitsplatz zu wechseln, wenn sie woanders ihre Vorstellungen besser verwirklichen können.

Dies heißt für die Träger, dass sie bei der Gewinnung und beim Einsatz von Mitarbeitenden immer öfter Kompromisse schließen müssen. Trotzdem müssen sie zugleich auch im Interesse der zu betreuenden Menschen weiterhin Flexibilität und Einsatzbereitschaft einfordern, was diesen Erwartungen der Mitarbeiter widerspricht!

\section{Wie man diesen Widerspruch auflösen kann}

Dies wird auf Dauer nur mit komplexen Maßnahmen möglich sein, indem man auch bereit ist, unkonventionelle Wege zu gehen und die Leistungserstellung grundsätzlich neu denkt. Ein wesentlicher Ansatz besteht darin, sich bei der Leistungserstellung vom heutigen Professionalisierungsgrad zu verabschieden. Ein Teil der Träger leistet sich heute den (beneidenswerten) Luxus, die Leistungen mit einem höheren Anteil an Fachkräften zu erbringen, als dies vertraglich oder organisatorisch erforderlich wäre. Hier können ohne Vertragsanpassungen mit den Kostenträgern durch eine feinere Steuerung noch Reserven erschlossen werden, indem der Anteil der Fachkräfte auf das erforderliche Maß reduziert wird und der freigewordene Anteil fürs Wachstum genutzt wird. Die Arbeiten müssen dann auf die (noch) verfügbaren Nichtfachkräfte delegiert werden.

In vielen Einrichtungen bewegt man sich jedoch schon am unteren Limit des vertraglich oder gesetzlich möglichen oder sogar darunter. Hier muss ernsthaft erwogen werden, wie durch eine andere Organisation mit einer geringeren Fachkraftquote die Leistungen erbracht werden können. Dies setzt auch eine Vertragsanpassung voraus, was jedoch zum Teil (z. B. stationäre 


\title{
Wir sind vorübergehend nicht im Dienstzimmer anzutreffen.
}

\section{$Z u$ den vereinbarten Zeiten stehen wir innen gerne wieder zur Verfügung. Danke für ihr Verständnis.}

\author{
Ihr Betreuungsteam
}

Insgesamt zeigt sich, dass die Ressource "Personal" heute viel differenzierter betrachtet werden muss, als dies in der Vergangenheit der Fall war. Einerseits ist der Einsatz von Fachkräften in vielen Fällen weiterhin zwingend erforderlich. Andererseits ist ein flächendeckender Einsatz von Fachkräften aus Kostengründen, aber auch aufgrund der Zergliederung der Hilfeleistungen gar nicht mehr möglich. Blickt man sich um, gibt es bereits Ideen und Lösungsansätze, die hier die Richtung weisen können:

- Stärkere Sozialraumorientierung und Schaffung von sogenannten "Circles of support«: Wenn öffentlich-refinanzierte hauptamtliche Strukturen nicht mehr ausreichen, um eine adäquate Leistungserbringung zu gewährleis- ten, müssen andere Akteure gewonnen und eingebunden werden. Neben Ehrenamtlichen sind hier Nachbarn, Freunde, Familie, aber auch Vereine, Pfarrgemeinden usw. denkbar.

- Stärkerer Technikeinsatz: Eine Entlastung hauptamtlicher Mitarbeitenden von administrativen Aufgaben kann beispielsweise durch den Einsatz einer mobilen Datenerfassung in den ambulanten Angeboten der Kinder- und Jugend- sowie Behindertenhilfe erreicht werden. Noch weiter geht das Konzept des "Ambient Assisted Living (AAL)", bei dem beispielsweise durch entsprechende Kommunikationsmittel der Personalanteil vor Ort und unproduktive Fahrzeiten reduziert werden können.
" "Ambulantisierung" der Arbeitsweisen in stationären Einrichtungen: Der Personaleinsatz in stationären Einrichtungen kann gezielter erfolgen, wenn er sich an dem im ambulanten Kontext orientiert. So gibt es in manchen Altenhilfeeinrichtungen beispielsweise "Pflegetouren" auf den Stationen mit Einsatzplan, fester Tour etc. - im Grunde ähnlich der ambulanten Pflege.

- Ambulant betreute Wohngemeinschaften: Hier werden auch vom (Bundes-) Gesetzgeber gewollt (aber nicht von allen Bundesländern in den Heimgesetzen unterstützt) bewusst Türen für alternative Versorgungsformen mit einer geringeren Regulationsintensität ohne starre Personalvorschriften geöffnet.

Die Fachkräfte müssen den zukünftigen Anforderungen flexibel begegnen und die anstehenden Veränderungen positiv mittragen und die neuen Rollen annehmen. Dies ist ein hehrer Anspruch, den die Arbeitgeber nicht als selbstverständlich erwarten dürfen, sondern durch Organisations- und Personalentwicklungsmaßnahmen befördern müssen. Dies erfordert erhebliche Innovationsbereitschaft und eine Neudefinition des Verhältnisses Betreuer und Betreute - oder: Dienstleister und Klient - und insbesondere der Funktion der sozialen Organisationen als Teil von Netzwerken.

Attila Nagy/Carsten Effert
Pflege) nur durch Gesetzesänderungen möglich wäre. In anderen Segmenten wie der Jugend- oder Eingliederungshilfe, aber auch in ambulanten Angeboten der Altenhilfe besteht jedoch die Möglichkeit, neue Angebote zu entwickeln, die andere Betreuungsquoten und Versorgungsformen umfassen. Für diese muss aktiv geworben und Lobbyarbeit betrieben werden - und zwar anders als bisher nicht mit der Forderung nach einem höheren Fachkräfteanteil, sondern nach einem niedrigeren!

Dem knappen Gut »Fachkräfte « kommt in diesem Kontext eine neue Aufgabe zu, indem sie selbst Netzwerke steuern, teilweise aufbauen und gestalten. Hierbei müssen sie zunehmend ehrenamtliche Kräfte, Angehörige oder andere Dienstleister einbinden. Diese Aufgabe liegt sicher nicht allen altgedienten Mitarbeitenden, kommt aber möglicherweise gerade den jüngeren Mitarbeitenden entgegen, die in vieler- lei Hinsicht ein partnerschaftliches Verständnis und ein Denken in Netzwerken aufweisen.

\section{Resümee}

Insgesamt zeigt sich, dass die Ressource »Personal« heute viel differenzierter betrachtet werden muss, als dies in der Vergangenheit der Fall war. Einerseits ist der Einsatz von Fachkräften in vielen Fällen weiterhin zwingend erforderlich. Andererseits ist ein flächendeckender Einsatz von Fachkräften aus Kostengründen, aber auch aufgrund der Zergliederung der Hilfeleistungen gar nicht mehr möglich. Blickt man sich um, gibt es bereits Ideen und Lösungsansätze, die hier die Richtung weisen können (vgl. Kasten).

Die Fachkräfte müssen den künftigen Anforderungen flexibel begegnen und die anstehenden Veränderungen positiv mittragen und die neuen Rol- len annehmen. Dies ist ein hehrer Anspruch, den die Arbeitgeber nicht als selbstverständlich erwarten dürfen, sondern durch Organisations- und Personalentwicklungsmaßnahmen befördern müssen. Dies erfordert erhebliche Innovationsbereitschaft und eine Neudefinition des Verhältnisses Betreuer und Betreute - oder: Dienstleister und Klient - und insbesondere der Funktion der sozialen Organisationen als Teil von Netzwerken.

Die erfolgreiche Bewältigung dieser Fragen dürfte vielleicht die größte Herausforderung für die Sozialwirtschaft der nächsten Jahrzehnte sein. 